\title{
Etiología de los defectos de desarrollo del esmalte. Revisión de la literatura
}

\author{
Etiology of developmental enamel defects as oral problem. Literature review \\ Mariana Ángeles-Vázquez ${ }^{a}$, Martha Mendoza-Rodríguez ${ }^{b}$ Carlo E. Medina-Solis ${ }^{c}$, Sara Celina \\ Conde-Pérez ${ }^{d}$, Miguel Ángel Fernández-Barrera ${ }^{e}$, Sonia Márquez-Rodríguez ${ }^{f}$
}

\begin{abstract}
:
Introduction: During the formation of enamel or amelogenesis, alterations that are identified as enamel defects can occur. These are commonly observed in child population, both in temporary and permanent eruption, from 6 months to 12 years. They are of multiple etiology.

Methodology: The electronic consultation was done through different search engines such as PubMed, Google Scholar, SciELO.

Results: A total of 20 research articles were found which met the criteria required for inclusion in this study.

Conclusion: Providing timely treatment to patients with developmental enamel defects (DED) is of utmost importance due these alterations have a great aesthetic, functional and psychological impact on children.
\end{abstract}

Keywords:

Developmental enamel defects, prevalence, etiological factors, caries

\section{Resumen:}

Introducción: Durante la formación del esmalte o amelogénesis pueden ocurrir alteraciones que se identificarán como defectos de esmalte (DDE). Estos son ampliamente observados la población infantil, tanto en la erupción temporaria como en la permanente, desde los 6 meses hasta los 12 años. Son de etiología múltiple.

Metodología: La consulta electrónica se hizo por medio de distintos buscadores como PubMed, Google Académico, SciELO.

Resultados: Se encontraron un total de 20 artículos de investigación los cuales cumplieron con los criterios requeridos para su inclusión en este estudio

Conclusiones: Dar tratamiento oportuno a los pacientes que presenten defectos del desarrollo del esmalte (DDE), es de suma importancia debido a que estas alteraciones tienen gran impacto estético, funcional y psicológico en la población infantil

Palabras Clave:

Defectos de desarrollo del esmalte, prevalencia, factores etiológicos, caries.

\section{Introducción}

El esmalte dental es la estructura mineralizada más dura del cuerpo humano, la cual presenta propiedades tanto físicas como químicas que lo hacen único. Está constituida en un $98 \%$ de minerales (en su mayoría hidroxiapatita) y menos del $2 \%$ de agua y materia orgánica. ${ }^{1}$ Es un tejido de origen ectodérmico y es sintetizado en el periodo de la formación del diente (odontogénesis) entre la sexta y séptima semana de

\footnotetext{
${ }^{a}$ Universidad Autónoma del Estado de Hidalgo, Instituto de Ciencias de la Salud, https://orcid.org/0000-0002-5456-7657,

Email: marianaanvaz5581@gmail.com

${ }^{\text {b }}$ Autor de Correspondencia, Universidad Autónoma del Estado de Hidalgo, Instituto de Ciencias de la Salud,

https://orcid.org/0000-0001-7887-7580,Email: martha_mendoza2138@uaeh.edu.mx

${ }^{c}$ Universidad Autónoma del Estado de Hidalgo, Instituto de Ciencias de la Salud, https://orcid.org/0000-0002-5456-7657, Email: cemedinas@yahoo.com

d Universidad Autónoma del Estado de Hidalgo, Instituto de Ciencias de la Salud, https://orcid.org/0000-0001-8313-1169, Email: lulumaco_1@yahoo.com.mx

${ }^{\text {e } U n i v e r s i d a d ~ A u t o ́ n o m a ~ d e l ~ E s t a d o ~ d e ~ H i d a l g o, ~ I n s t i t u t o ~ d e ~ C i e n c i a s ~ d e ~ l a ~ S a l u d, ~ h t t p s: / / o r c i d . o r g / 0000-0001-7176-9587, ~ E m a i l: ~ m a f b a . m a f b @ ~ g m a i l . c o m ~}$

${ }^{\mathrm{f}}$ Universidad Autónoma del Estado de Hidalgo, Instituto de Ciencias de la Salud, https://orcid.org/0000-0003-1769-8276, Email: cdsoniamr@hotmail.com

${ }^{g}$ Universidad Autónoma del Estado de Hidalgo, Instituto de Ciencias de la Salud, https://orcid.org/0000-0001-7972-1406, Email: araccondep@ @otmail.com
} 
gestación y continúa durante años después del nacimiento. ${ }^{2}$

El periodo de formación del esmalte es conocido como amelogénesis y cualquier defecto durante este proceso provoca cambios irreversibles, puesto que el ameloblasto (células encargadas de la formación del esmalte), tiene poca capacidad para regenerarse ${ }^{3}$, por lo tanto, la apariencia clínica de los defectos de desarrollo del esmalte (DDE) se relacionan con la etapa de formación del esmalte en la que se produce la anomalía, con la intensidad y duración del agente agresor. ${ }^{4}$

En los DDE son visibles desviaciones de la apariencia translúcida normal del esmalte dental, resultante de la disfunción del órgano del esmalte. Estas podrían ser de naturaleza cuantitativa, que se manifiesta como una deficiencia en el espesor del esmalte o hipoplasia del esmalte (HE), o cualitativa (hipomineralización), presentándose clínicamente como opacidad del esmalte (OE). Estos DDE pueden tener un importante impacto negativo en la salud bucal y la estética, como por ejemplo susceptibilidad a la caries, un mayor desgaste dental, sensibilidad y una percepción estética menos favorable. ${ }^{5}$ El objetivo de este trabajo es conocer los distintos factores etiológicos de los defectos de desarrollo del esmalte, así como también el impacto que tienen los diversos factores en la salud bucodental de la población en general.

\section{Material y métodos}

Esta revisión de la literatura fue obtenida mediante la búsqueda electrónica de diversas plataformas, PubMed, Google Académico, SciELO utilizando palabras como "Defectos de desarrollo del esmalte en dentición mixta", "Developmental Enamel Defects", "Enamel Defects" "Hipomineralización", "Opacidades del esmalte" "Dental Fluoosis", "Enamel Hipoplasia" se seleccionaron manualmente los documentos relevantes de acuerdo al perfil de esta revisión.

La búsqueda sistemática se limitó a artículos publicados entre el 2007-2019 sin hacer discriminación por idioma de publicación.

\section{Resultados}

Se encontraron un total de 44 artículos, de los cuales solo 20 cumplieron con los criterios necesarios para poder ser incluidos en la presente investigación

\section{Discusiones}

Para entender mejor el proceso de formación del esmalte, es necesario tener claras ciertas generalidades acerca de la odontogénesis. Diversos autores coinciden en la descripción de los fenómenos que ocurren en cada etapa de la formación del diente $e^{6,7,1,8,9}$. Se consideraron que los eventos más relevantes de cada etapa fueron los siguientes:

- Etapa de yema o brote: Etapa en la cual se observa que, en la lámina dental de cada diente, hay un abultamiento en forma de disco, que serán lo que se conoce como brote o yemas epiteliales. El mesénquima subyacente en contacto con la yema presenta una condensación esférica de células mesenquimatosas que evolucionará para constituir la papila dental.

- Etapa de casquete: Quedan diferenciadas estructuras como el órgano dental epitelial, la papila dental y el saco dental, responsables de la formación de todos los tejidos del diente y del tejido correspondiente al periodonto. Empieza la histodiferenciación del órgano dental. En su parte cóncava se forma el epitelio adamantino interno en el cual las células cuboides se transforman en cilíndricas y en la porción convexa del casquete, se forma el epitelio adamantino externo en el cual las células cuboides no cambian su forma, además y el retículo estrellado a consecuencia de la segregación de glicosaminoglicanos por las células poliédricas centrales del órgano dental ${ }^{7,8}$.

- Etapa de campana: en esta etapa se establecen los patrones coronarios de cúspides bordes y fisuras. Se desarrolla el estrato intermedio entre el retículo estrellado y el epitelio adamantino interno el cual es esencial en la formación del esmalte al producirse los materiales que pasan a los ameloblastos y a la matriz del esmalte durante la amelogénesis. El retículo estrellado se expande por aumento de la sustancia intercelular. Al final de esta etapa el epitelio adamantino externo se dispone en pliegues en los que penetran proyecciones del saco dental que proporcionan vasos capilares al órgano del esmalte durante la amelogénesis. Se produce la diferenciación de los ameloblastos y de los odontoblastos. Por la influencia organizadora de las células del epitelio adamantino interno, las células de la papila dentaria se diferencian en odontoblastos, mientras que las células cilíndricas de este epitelio, originarán a los ameloblastos. La papila dental en su evolución posterior formará la dentina y la pulpa. El saco dental adopta forma circular y formará al cemento, al ligamento periodontal y al hueso alveolar propio ${ }^{7,8}$.

- En el último estadío se pierde la continuación del órgano dental donde la lámina y el saco dental rodeará completamente al germen dentario. 


\section{Hidalgo, Vol. 8, No. 16 (2020) 187-193}

- Cuando la diferenciación de los tejidos del germen alcanza su nivel máximo se inicia la formación de los tejidos mineralizados. Esta nueva etapa se reconoce por numerosos autores como etapa de folículo dentario, aunque otros solo la consideran como una etapa avanzada de la campana ${ }^{6,7}$.

En la literatura revisada son numerosos los autores que coinciden con los acontecimientos que suceden durante la amelogénesis y es necesario enfatizar en ellos para poder entender mejor el origen de los $\mathrm{DDE}^{7,3,10}$.

Partiendo de que la formación de los tejidos mineralizados se inicia en la zona de las cúspides y bordes incisales y que es la dentina el primer tejido dentario que se forma, se describen estos acontecimientos teniendo en cuenta aquellos donde más coincidencia se encontró durante la revisión realizada.

En la etapa de folículo dentario el epitelio adamantino interno muestra una intensa actividad citogenética en esta etapa y está separado de la papila dental por la lámina basal, cuyo límite será la futura unión amelodentinaria.

Las células del epitelio externo del órgano dental, se vuelven irregulares y en su lado convexo aparecen pliegues en el interior de los cuales penetran capilares del saco dental, que asegurarán el aporte nutricional al órgano dentario en las etapas sucesivas al detenerse el aporte de la papila dental cuando se forman las primeras capas de dentina.

Previa a la diferenciación completa de los ameloblastos, estas células en interacción con las adyacentes de la papila determinan la forma del límite amelodentinario y de la corona del diente a la vez que ocasionan la diferenciación de las células de la papila en odontoblastos y ocurre la formación de las primeras capas de dentina. Consecutivamente los capilares del saco dentario proliferan y el retículo estrellado reduce su tamaño, lo que acorta la distancia entre los vasos y el epitelio interno del órgano dental.

Luego de formadas las primeras capas de dentina se inicia la secreción de la matriz del esmalte. En el polo secretorio de los ameloblastos se concentran numerosas vesículas cuyo contenido se segrega y forma la matriz orgánica del esmalte. La primera matriz que se deposita forma una capa delgada en contacto con la dentina y recibe el nombre de membrana dentinoesmáltica.

Luego de la formación de la membrana dentinoesmáltica, la matriz se deposita delineando una proyección del ameloblasto conocida como proceso de Tomes, a través del cual se continúa la secreción del esmalte.

A medida que se forma la matriz, los amelobalstos se desplazan hacia afuera en dirección al epitelio externo, hasta formar el total del esmalte dentario.
Coincidentemente con la deposición de la matriz aparecen dentro de ella los cristales de hidroxiapatita que al parecer son segregados por las vesículas del polo secreto del ameloblasto, ello explica que no se pueda apreciar una zona de matriz sin calcificar como ocurre en los otros tejidos mineralizados del diente.

La calcificación de la matriz del esmalte se divide en tres etapas, la impregnación por estratos que es casi simultánea con la formación de la matriz y determina la impregnación de esta con 25 o 30 \% de la masa total de sales que debe contener el esmalte. Este proceso marcha con cierto retraso con respecto a la formación de la matriz de manera que siempre queda una fina capa más profunda, vecina a límite amelodentario que son las más antiguas y más calcificadas con respecto a las más superficiales que no han recibido sales o recién comienza a recibirlas, o sea, esta primera fracción de las sales de calcio se deposita en estratos siguiendo la misma dirección en que se ha depositado la matriz. La impregnación en masa donde le llega el 60 o 70 \% de su masa total de sales con lo que se completa el 93 o $95 \%$ de sustancia inorgánica que posee el esmalte maduro.

En esta etapa las sales no se depositan en capas, sino en forma masiva y se distribuyen homogéneamente por toda la matriz orgánica, las sales se mantienen en estado coloidal, esta impregnación comienza por las cúspides y progresa hacia el cuello en planos aproximadamente perpendiculares a las líneas de Retzius. La última etapa es la cristalización durante todo este período las sales de calcio se movilizan al estado de solución o de compuestos orgánicos coloides. Recién cuando se ha completado la afluencia de sales inorgánicas se produce su cristalización, se inicia en la superficie de las cúspides o bordes incisales y progresa hacia la zona cervical. Para la impregnación de las sales de calcio en la sustancia orgánica es necesario una gran proporción de agua, la cristalización requiere que gran parte de esa sustancia orgánica y agua sean nuevamente eliminadas. Se considera que la consistencia cartilaginosa del esmalte inmaduro está dada por la matriz orgánica que en este período es insoluble a los ácidos y radiotraslúcida. Después de la cristalización el esmalte pierde agua, se vuelve duro y se hace soluble a los ácidos. ${ }^{7}$

\section{Clasificación de los defectos de desarrollo del esmalte}

Para la clasificación de los defectos del desarrollo del esmalte se utiliza el Índice de Defectos de Desarrollo del Esmalte Modificado (Índice DDEm) el cual se utiliza en estudios epidemiológicos por su simplicidad y criterios, los cuales cubren la mayoría de defectos de desarrollo del esmalte, como a continuación se describe ${ }^{4}$ : 
Hipoplasia: Anomalía que involucra la superficie del esmalte y se asocia con un espesor reducido y localizado, puede aparecer en forma de: a) fosas únicas o múltiples, superficiales $o$ profundas, aisladas $u$ organizadas horizontalmente a través de la superficie dental; b) surcos simples o múltiples, estrechos o amplios (máximo $2 \mathrm{~mm}$ ), o ausencia parcial o total del esmalte sobre un área considerable de dentina. El esmalte de espesor reducido puede ser traslucido u opaco.

Opacidad demarcada: anomalía que afecta la traslucidez del esmalte en grado variable. El esmalte dañado es de espesor normal con una superficie lisa Es fácilmente distinguible de un esmalte normal adyacente y puede ser de color blanco, crema, amarillo o café. Las lesiones varían en extensión, localización y distribución en la boca. Algunas lesiones mantienen una superficie traslúcida, mientras otras se ven clínicamente mate.

Opacidad difusa: defecto que involucra una alteración en la traslucidez del esmalte en grado variable. El esmalte afectado es de espesor normal y en la erupción tiene una superficie relativamente suave y su color es blanco. No hay nitidez en los límites del esmalte sano adyacente con el afectado y puede ser de distribución:

Lineal: Líneas blancas de opacidad que siguen las líneas de desarrollo de los dientes. Puede ocurrir confluencia de líneas adyacentes.

Parche: Áreas nubosas irregulares de opacidad sin márgenes bien definidos.

Confluente: Irregularidades difusas que se confunden en un área blanca tiza, que se extiende de mesial a distal, cobren toda la superficie o están confinadas a un área localizada.

Parche confluente más pigmentación o pérdida de esmalte: Cambios posteruptivos de color o pérdida de esmalte relacionada solo con áreas hipomineralizadas. Por ejemplo: apariencia de pérdida en fosas o áreas amplias de esmalte rodeadas por esmalte blanco tiza o pigmentado 4 .

En 1982 la FDI estableció un índice para evaluar de DDE, el cual incluyó las siguientes categorías:

- Tipo de defecto, con dos opciones para opacidades discriminadas por color (blanco/amarillo), cuatro opciones para hipoplasias, una para esmalte decolorado, una para otros defectos y una para combinación de defectos.
- Número y demarcación de los defectos, con cuatro opciones (simple, múltiple, líneas blancas finas difusas, parche difuso).

- Localización del defecto, con siete opciones. • Necesidad de tratamiento, con seis opciones.

- Historia médica, con cuatro opciones.

- Historia dental, con seis opciones.

- Etiología de los DDE, con cinco opciones.

En 1992, la FDI (Federación Dental Internacional) publicó una versión modificada del Índice DDE (Índice de Desarrollo del Esmalte modificado: IDDEm ), el cual no era muy diferente del planteado por Clarkson y O'Mullane en $1982^{11}$, volvieron a clasificar los DDE en base a su apariencia clínica; asignaron color solo a las opacidades demarcadas, puesto que la alteración de color en las opacidades difusas era secundaria; ampliaron el rango para describir más subcategorías de opacidades difusas, redujeron las subcategorías de hipoplasias, quitaron el término decoloración, y en su lugar dejaron la categoría otros defectos, permitieron el registro de combinaciones de defectos en códigos adicionales, incluyeron el registro de la extensión del defecto ${ }^{4}$. Así como se observa en la Tabla 1.

Tabla. 1 Índice DDE modificado 4.

\begin{tabular}{|l|l|}
\hline Categorías de DDE & Código \\
\hline Normal & 0 \\
\hline Opacidad demarcada & \\
\hline Blanco/crema & 1 \\
\hline Amarillo/marrón & 2 \\
\hline Opacidad difusa & \\
\hline Lineal & 3 \\
\hline Parche & 4 \\
\hline Confluente & 5 \\
\hline $\begin{array}{l}\text { Confluente/parche+pigmentación+ } \\
\text { pérdida de esmalte }\end{array}$ & 6 \\
\hline Hipoplasia & \\
\hline Fosas & \\
\hline Con ausencia total de esmalte & 8 \\
\hline Cualquier otro defecto & 9 \\
\hline Combinaciones & \\
\hline Demarcada y difusa & A \\
\hline Demarcada e hipoplasia & B \\
\hline Difusa e hipoplasia & C \\
\hline Los tres defectos & D \\
\hline
\end{tabular}


EI IDDEm se complementa con la extensión de la lesión, determinando así la severidad del problema mencionan las siguientes extensiones:

- $\quad$ Normal: Identificada con el código 0.

- Menos de 1/3 del diente: Identificada con el código 1 Fig. 1

- $\quad$ De 1/3 a 2/3 del diente: Identificada con el código 2 Fig. 2

- Más de 2/3 del diente: Identificada con el código 3 Fig.3

Esto facilita la evaluación de DDE y por lo tanto la elección del plan de tratamiento, es por esto que la clasificación de Índice de desarrollo del esmalte adoptó este apartado.

Cuando se elige la extensión que abarca el defecto del esmalte se está dando la severidad de la lesión ${ }^{3}$.

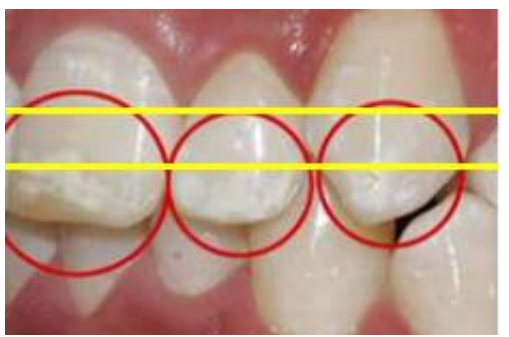

Fig. 1 Extensión de $1 / 3$ en el esmalte ${ }^{6}$

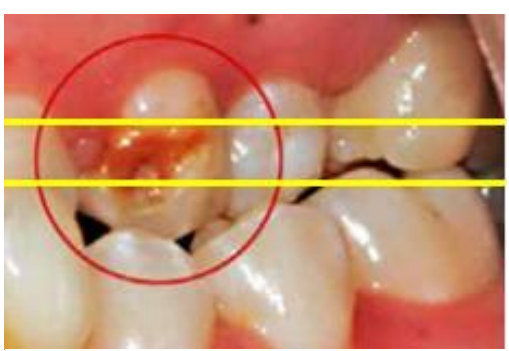

Fig. 2 Extensión de 1/3 a 2/3 en el esmalte $^{6}$

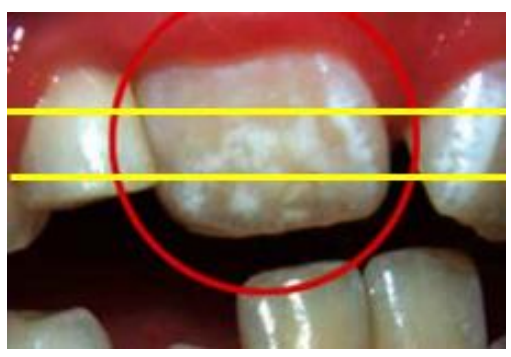

Fig. 3 Extensión de más de 2/3 en el esmalte $^{6}$

\section{Etiología}

La etiología de los defectos del desarrollo del esmalte es poco definida, en diversas investigaciones mencionan a los factores ambientales locales o sistémicos, causas genéticas o bien una interacción de ambos como los causantes de alterar el proceso metabólico de los ameloblastos en la formación del esmalte, produciendo $\mathrm{DDE}^{12,13,14}$.El momento de acción de estos factores puede ser en el proceso de gestación, durante la formación ontogénesis o después del nacimiento, de tal manera que pueden presentar afección la dentición primaria o secundaria 0 estar afectadas ambas denticiones. Generalmente los dientes permanentes presentan mayor afección, la apariencia clínica que se observará en el diente dependerá del estadío de formación y la agresión del factor etiológico ${ }^{13}$.

Reportes de diversos estudios acerca de DDE asocian estas anomalías a niños que presentan problemas renales crónicos, fibrosis quística, bajo peso al nacer, padres diabéticos, enfermedad celiaca ${ }^{15}$, labio y/o paladar hendido ${ }^{16}$, leucemia linfoblástica ${ }^{17}$, parálisis cerebral, síndrome de Down ${ }^{18}$, niños pre términos o de bajo peso al nacer ${ }^{1}$. Se reporta que son aproximadamente 100 agentes etiológicos los causantes de la alteración en la formación del esmalte en diferentes $\operatorname{grados}^{19}$.

\section{Condiciones adquiridas}

Numerosas condiciones tanto sistémicas, como locales adquiridas durante los periodos prenatal, perinatal y postnatal de desarrollo pueden causar daño en el desarrollo del esmalte y resultar en DDE en la dentición primaria $^{20}$.

Las condiciones prenatales que han sido asociadas con la hipoplasia del esmalte en niños incluyen, deficiencia de vitamina $D$ durante embarazo, tétanos neonatal, si la madre fumó durante el embarazo, aumento excesivo de la madre durante el embarazo sin ningún tipo de atención médica $^{20}$.

Múltiples nacimientos también es un factor de riesgo para los DDE debido al alto índice de complicaciones neonatales que experimentan este tipo de niños. A pesar de que no es muy común encontrar este tipo de problemas en países desarrollados, las deficiencias nutricionales en los niños, particularmente aquellas asociadas a la absorción insuficiente de Vitaminas A, C, $D$ y calcio son conocidos como factores de riesgo de la hipoplasia del esmalte. También, la nutrición extendida a base de leche materna sin ser complementada con alimentos sólidos también ha sido sugerida como una causante de DDE en dentición primaria ${ }^{20}$.

Niños que han nacido de forma prematura o con bajo o muy bajo peso al nacer tienen mayor prevalencia de hipoplasias en el esmalte que niños nacidos a término 
con peso normal. Hay anormalidades asociadas con la mineralización, así como la hipocalcemia, osteopenia e hiperbilirrubinemia, han sido directamente implicadas en la etiología de los DDE. Enfermedades renales y hepáticas también han sido asociadas con hipoplasia del esmalte; así como también enfermedades infecciosas causadas por virus y bacterias, tales como infecciones del tracto urinario, otitis e infecciones de vías respiratorias altas; sífilis congénita adquirida por medio de la madre, Treponema pallidum e infecciones virales como varicela, rubeola, sarampión, paperas e influenza también han sido relacionadas con los DDE en ambas denticiones ${ }^{20}$.

En muchos niños con parálisis cerebral es común observar DDE y son causados por disturbios sistémicos los cuales han dañado las células de formación neurológicas y del esmalte.

Diversos fármacos tienen potencial para dañar a los ameloblastos y causar DDE; por ejemplo, niños con exposiciones elevadas a productos que contienen plomo o ingesta accidental de estos reportan hipoplasia del esmalte. Las tetraciclinas son bien conocidas por causar pigmentaciones dentales e hipoplasia del esmalte. Más recientemente, la Amoxicilina ha sido implicada como causante de hipoplasia, aunque tampoco se pueden descartar los efectos de las fiebres e infecciones los cuales requieren el uso de antibióticos para su tratamiento ${ }^{20}$.

\section{Condiciones hereditarias}

Los defectos del esmalte pueden ser solo características en condiciones hereditarias que envuelven únicamente al esmalte dental o pueden ser un componente de un síndrome sistémico generalizado. Las condiciones hereditarias que envuelven únicamente al esmalte son conocidas como amelogénesis imperfecta y los defectos se pueden presentar como hipoplasia del esmalte, hipomineralización o hipomaduración. Las anormalidades de los genes involucrados en la amelogénesis principalmente son los responsables de estos defectos. En niños con amelogénesis imperfecta, característicamente, los dientes tanto en dentición permanente como en dentición decidua presentan DDE. Existen muchos síndromes hereditarios los cuales tienen como característica hipoplasia del esmalte, como por ejemplo en el síndrome de Usher, el cual se caracteriza por la pérdida auditiva neurosensorial, retinitis pigmentosa e hipoplasia del esmalte; así como también el síndrome de Seckel que se caracteriza por discapacidad intelectual múltiples defectos esqueletales. Síndrome de Ellis Van Creveld, el cual también presenta hipoplasia del esmalte junto con defectos esqueletales y cardiacos. Los DDE también han sido asociados con el síndrome de Treacher Collins, síndrome otodental, síndrome velocardiofacial y síndrome de Heimler ${ }^{20}$.
En diversos estudios han relacionado los defectos de desarrollo del esmalte con caries, fracturas dentales e hipersensibildad ${ }^{5,12}$; lo que hace de estas anomalías un problema frecuente en la población. En una publicación realizada en 2013 por Sierra y cols. excluyen hipoplasia del resto de los DDE y relacionan estas anomalías con los mismos factores mencionados por Mafla y cols. (caries, fracturas).

Hay múltiples factores que han sido relacionados con la aparición de DDE, genéticos, ambientales, nutricionales, entre muchos otros. Todos causantes de alterar el proceso metabólico de los ameloblastos en la formación del esmalte ${ }^{12,13,14}$.

Niños prematuros y con bajo peso al nacer han sido correlacionados con los defectos desarrollo del esmalte ${ }^{1}$. Seow y cols. coinciden con lo planteado por Krishnaji y cols. en un estudio de etiología de DDE realizado en $2019^{20}$.

\section{Conclusión}

La prevalencia los defectos de desarrollo del esmalte es muy elevada, son un problema muy común que afecta ambas denticiones, este tipo de alteraciones hacen a los órganos dentales más propensos a tener caries, fracturas dentales e hipersensibilidad. Debido a esto es de suma importancia educar a la población para que tenga conocimiento de estas anomalías y de esta manera puedan ser detectadas a tiempo, y así poder dar tratamiento oportuno.

En la actualidad existe una gran gama de tratamientos como carillas, resinas coronas, los cuales tienen como objetivo devolver la funcionalidad y la estética de los órganos dentales.

\section{Referencias}

1. Krishnaji Musale, P., Shrikant Soni, A., \& Sunil Kothare, S. (2019). Etiology and Considerations of Developmental Enamel Defects in Children : A Narrative. Journal Pediatric Review, 141-150.

2. Pedroso Ramos, L., Reyes Suárez, V. O., González Rodríguez, S., \& Boizán Soler, D. (2019). Anomalías estructurales del esmalte y afectación estética en escolares de 6-17 años de Cojímar. Revista Electrónica Medimay, 4-13.

3. S Lacruz, R., Habelitz J, S., Wright, T., \& L Paine, M. (2017). Dental Enamel Formation and Implications for Oral Health and Disease. American Physiological Society, 939-993.

4. Sierra., M. C. (2013). Terminology, Classification and Measuring of the Devel opmental Def ects. Univ Odontol, 3344.

5. Mafla, A. C., Córdoba Urbano, D. L., Rojas Caicedo, N. M., Vallejos de la Rosa, M. A., Erazo Sánchez, M. F., \& Rodríguez Caicedo, J. (2014). Prevalencia de defectos del esmlate dental en niños y adolescentes colombianos. Facultad de Odontología Universidad de Antioquia, 106-125. 
6. Albertí Vázquez, L., Más Sarabia, M., Martínez Padilla, S., \& Méndez Martínez , J. (2007). Histogénesis del esmalte dentario. Consideraciones generales. Archivo médico de Camagüey, 5061.

7. Gómez de Ferraris, M. E., \& Campos Muñoz, A. (2009) Histología, Embriología e Ingeniería Tisular Bucodental. Panaméricana.

8. Masumo, R., Bardsen, A., \& Nordrehaug, A. (2013) Developmental defects of enamel in primary teethand association with early life course events: a study of 6-36 month old children in Manyara, Tanzania. BMC Oral Health, 13-21

9. Vargas Ferreira , F., \& Machado Ardengui, T. (2011). Developmental enamel defects and their impact on child oral health-related quality life. Braz Oral Res, 531-537.

10. Pedroso Ramos, L., Gonzáles Rodríguez, S., \& Reyes Suárez, V. O. (2015). Anomalías estructurales del esmalte y afectación estética en escolares de 6-17 años de Cojimar. Obtenido de http://www.estomatologia2015.sld.cu/index.php/estomatologia/ nov2015/paper/view/799

11. Clarkson, J., \& O’Mullane, D. (1989). A Modified DDE Index for Use in Epidemiological Studies of Enamel Defects. Journal of Dental Research, 445-450.

12. Vallejos Sánchez, A., Medina Solis, C. E., Casanova Rosado, J., Minaya Sánchez, M., Robles Minaya, J., \& De la Rosa Santillana, R. (2012). Prevalencia y distribución de los defectos de desarrollo del esmalte en dentición permanente de escolares de 6 a 12 años. Revista Latinoamericana de ortodoncia y Odontopediatria, 1-9.

13. Camargo, M. G. (2010). Defectos de esmalte en la población infantil. ODOUS CIENTIFICA, 50-57.

14. Taddei-Moran, F. (2012). Anomalias del esmalte dental en una población peruana. Kiru, 131-135.

15. Sigala Robles, S., Aguayo Patrón, S. V., \& Calderón de la Barca, A. M. (2018). Genética, ambiente y asma asociados a enfermedad celiaca en la familia extendida de un niño afectado. Revista de Gastroenerología de México, 79-85.

16. Salas C, M. E., Barrios G, Z., Simancas, Y., Ablan, L., Ramírez , P., \& Prato, R. (2015). Anomalias dentarias en niños con fisura labio palatina. Revista odontológica de los Andes, 4-9.

17. Furtado Valdao, A., Gongalves de Motta, P., De Oliviera Dias, I., Vidigal Martins , I. C., Oliveira Figueiredo, I., \& Valiente de Oliviera, K. K. (2015). Manifestações orais nas doenças hematológicas: Revisão de literatura. Revista de Universidade Vale Rio Verde, Três Corações, 216-235.

18. Diéguez Pérez, M., Nova García, M. J., Mourelle Martínez, M R., \& Bartolomé Villar, B. (2016). Oral health in children with physical (Cerebral Palsy) and intellectual (Down Syndrome) disabilities: Systematic review I. Journal of Clinical and Experimental Dentistry, 337-343.

19. Taddei-Moran, F. (2012). ANOMALÍAS DEL ESMALTE DENTARIO EN NIÑOS DE 5 A 8 AÑOS DE. Kiru, 131-135.

20. Salanitri , S., \& Seow, K. (2013). Developmental eamel defects in the primary dentition: aetiology and clinical management Australian Dental Journal , 133-140. 